\title{
Identification and preservation of stained non-sentinel lymph nodes in breast cancer
}

\author{
XIAOYAN LI ${ }^{1}$, SISI CHEN ${ }^{1}$, YI DUAN ${ }^{1}$, HANYIN GUO $^{1}$, LIYU JIANG ${ }^{1}$, \\ XIAOLI KONG ${ }^{1}$, TINGTING MA ${ }^{1}$ and QIFENG YANG ${ }^{1,2}$ \\ Departments of ${ }^{1}$ Breast Surgery and ${ }^{2}$ Pathology Tissue Bank, Qilu Hospital, \\ Shandong University, Jinan, Shandong 250012, P.R. China
}

Received June 14, 2020; Accepted September 11, 2020

DOI: $10.3892 / \mathrm{ol} .2020 .12236$

\begin{abstract}
Sentinel lymph nodes (SLNs) are the first lymph nodes that receive lymphatic drainage from the breast. However, all stained lymph nodes are dissected as SLNs during surgery. The present study aimed to identify and preserve the stained non-SLNs and evaluate the safety during sentinel lymph node biopsy (SLNB) in breast cancer. SLNB was performed with a methylene blue and indocyanine green double-tracer technique. The first lymph node, which was connected with lymphatic vessels from the breast, was designated as the true SLN. The lymph node that was directly connected with the output lymphatic duct of the SLN was defined as post-SLN (poSLN), whereas the stained poSLN was designated as non-SLN. Both the stained SLN and non-SLN were sent to the pathological department for definitive diagnosis. The present study demonstrated that intraoperative dissection of the lymphatic network could distinguish true SLNs and stained non-SLNs. The number of stained lymph nodes was time-dependent. Not all stained lymph nodes were real SLNs, whereas the poSLNs would be stained if the staining time interval was inappropriate. The data indicated that the poSLNs were negative for metastasis when the SLNs were negative for metastasis. Stained lymph nodes may contain non-SLNs in addition to SLNs. Resection of all stained lymph nodes is not recommended. To reduce the morbidity due to SLNB complications, the identification and preservation of stained non-SLNs during SLNB is feasible and warrants further study in the era of precision medicine.
\end{abstract}

Correspondence to: Professor Qifeng Yang, Department of Breast Surgery, Qilu Hospital, Shandong University, 107 Wenhua Xi Road, Jinan, Shandong 250012, P.R. China

E-mail: qifengy_sdu@163.com

Key words: sentinel lymph node biopsy, post-sentinel lymph node, stained lymph nodes, breast cancer

\section{Introduction}

Sentinel lymph node biopsy (SLNB) was introduced in the 1990s (1). Sentinel lymph nodes (SLNs) are the first lymph nodes that receive lymphatic drainage from the breast (2-4). Due to the relative novelty of SLNB in surgical operations, definitive standards are not available. The number of removed SLNs that can accurately predict lymph node status remains controversial. Yi et al (5) observed that the removal of five SLNs was sufficient to identify the presence of metastatic lymph nodes for $>99 \%$ of patients. Kim et al (6) reported that the removal of at least two lymph nodes during SLNB may be acceptable. Boughey et al (7) and Boileau et al (8) reported that the removal of at least two sentinel nodes could lower the false-negative rate of SLNs in post-neoadjuvant chemotherapy. Yi et al (5) and Zakaria et al (9) reported that positive nodes were usually identified following the resection of the first four or five nodes. The German Gynecological Oncology Group recommends removal of at least two SLNs to reduce false negative rates (10). In order to reduce false negative rates and increase the detection rate, several surgeons may remove additional lymph nodes that are not true SLNs $(11,12)$. Sugie et al $(11)$ and Inoue et al (12) have published data on the removal of enlarged lymph nodes that are hard, palpable and suspicious for metastases, and can therefore be considered SLNs.

SLNs are theoretically the first lymph nodes reached by metastatic cancer cells that migrate from a primary tumor to the breast (13). SLNs are detected by injecting a dye into the breast and detecting the first draining lymph node(s), which is/are subsequently sampled as pathological specimens. In routine clinical practice, it has been reported that blue-stained and/or fluorescent-stained lymph nodes are typically defined as SLNs (12,14-17). The number of stained lymph nodes is time-dependent. However, the time intervals between observation and injection have not been uniformly reported in the literature. The time interval varies between 2 and $15 \mathrm{~min}(12,16,18-22)$. When SLNB was performed $2 \mathrm{~min}$ following injection, it was reported that the median number of SLNs was 1.8 per patient (range, 1-4) (19). The median number of SLNs was 2.4 (range, 1-7) when the time interval was 3.5-5.0 $\mathrm{min}$ (12). When SLNB was performed 5-15 min following injection, the median number of SLNs ranged between 1 and $9(16,22)$. Based on these studies, it is assumed 
that the number of SLNs performed by different medical practitioners may vary and that not all stained lymph nodes are true SLNs.

In the present study, SLNB was performed with methylene blue (MB) and the indocyanine green (ICG) double-tracer technique, and all the stained lymph nodes were observed carefully. The association between stained lymph nodes and the lymphatic draining network was clarified. The current report aimed to distinguish stained SNLs and stained non-SLNs. Furthermore, the assessment of the identification and preservation of stained resected non-SLNs during SLNB was performed in breast cancer.

\section{Materials and methods}

Patients. A total of 61 female participants were included in the present study. The median age of the patients was 49.44 years (range, 21-68 years). The patients were diagnosed with breast cancer between September 2016 and September 2018 at the Qilu Hospital, Shandong University (Jinan, China). Patients with clinical T1-T3N0M0 breast cancer (23) were considered eligible. The exclusion criteria included inflammatory breast cancer and stage IV disease (23). The axillary lymph node status was evaluated prior to surgery. All the patients received precise SLNB. Written informed consent was obtained from all patients, and the present study was approved by the Ethics Committee on the Scientific Research of Shandong University Qilu Hospital (Jinan, China; approval no. KYLL-2016-231).

Precise SLNB. Precise SLNB was performed for all the patients as previously described (24). Initially, MB and ICG were injected into the periareolar tissue. Subsequently, the lymphatic ducts were marked on the skin and the lymphatic channels were dissected precisely. All the stained lymph nodes were identified. All the first lymph nodes that received lymphatic drainage of the breast were designated as SLNs. The remaining stained lymph nodes were denoted as stained non-SLNs. The stained lymph nodes that directly followed the output lymphatic duct of the SLNs were defined as post-SLNs (poSLNs). True SLNs were stained during SLNB (Fig. 1A). In addition, the poSLNs could be stained if the time intervals were longer (Fig. 1B). The time from the injection to the dissection of the SLN and poSLN was $\sim 5-10 \mathrm{~min}$ and the SLNB was guided by the ICG fluorescence real-time dynamic imaging system (Jinan Smart Technology Co., Ltd.). The lymphatic vessels were observed transcutaneously in real time on the monitor screen.

Data collection. The SLNs and poSLNs were marked separately prior to histopathological examination, performed as previously described $(25,26)$. Intraoperative, frozen-section staining was performed with hematoxylin and eosin according to routine hospital procedures. The remaining SLN and poSLN tissues were formalin-fixed and paraffin-embedded for routine histopathological examination. Briefly, the tissues were harvested and fixed in $10 \%$ formalin at $24^{\circ} \mathrm{C}$ for $12 \mathrm{~h}$. The paraffin-embedded specimens were cut into $4-\mu \mathrm{m}$-thick sections and heated at $60^{\circ} \mathrm{C}$ for $60 \mathrm{~min}$. The status of SLNs and poSLNs was calculated by comparison of the results of the intraoperative frozen-section staining and by routine histopathological examination. If there were no tumor cells in
Table I. Characteristics of the patients with breast cancer $(n=61)$.

\begin{tabular}{lc}
\hline Variable & Total, $\mathrm{n}$ \\
\hline Age, years & \\
$\leq 45$ & 20 \\
$>45$ & 41 \\
Number of tumors & \\
1 & 55 \\
2 & 4 \\
3 & 1 \\
4 & 1 \\
Tumor size, cm & \\
$\leq 2$ & 38 \\
$>2$ & 23 \\
ER status & \\
Negative & 13 \\
Positive & 48 \\
PR status & \\
Negative & 12 \\
Positive & 49 \\
HER-2 status & \\
Negative & \\
Positive & 49 \\
\hline
\end{tabular}

ER, estrogen receptor; PR, progesterone receptor; HER-2, human epidermal growth factor receptor-2.

lymph nodes, the status was defined as negative. If there were tumor cells in lymph nodes, it was defined as positive.

\section{Results}

Patient information. Between September 2016 and September 2018, a total of 61 patients with breast cancer were enrolled. A total of 51 patients presented with invasive ductal cancer, 7 exhibited ductal carcinoma in situ, 1 had invasive lobular carcinoma and 2 exhibited another type of carcinoma. A total of 28 patients received axillary lymph node dissection (ALND) and the other 33 patients underwent mastectomy and SLNB.

SLNB with poSLN dissection in patients with breast cancer. The data demonstrated staining of certain lymph nodes that were identified subsequently to the SLNs. This staining occurred during precise SLNB and this type of lymph node was defined as a stained non-SLN. The lymph nodes that directly followed the SLNs were designated as poSLNs (Fig. 2). It has been suggested that all blue-stained nodes and/or fluorescence-positive lymph nodes can be removed and defined as SLNs (27). However, it was identified that the poSLNs could also be stained during the operation if the time interval following MB and ICG injection was too long. The exact methodology of SLNB and the decisions as to whether poSLNs should be dissected remain controversial. The present study was conducted to determine whether poSLNs should be dissected during SLNB. 

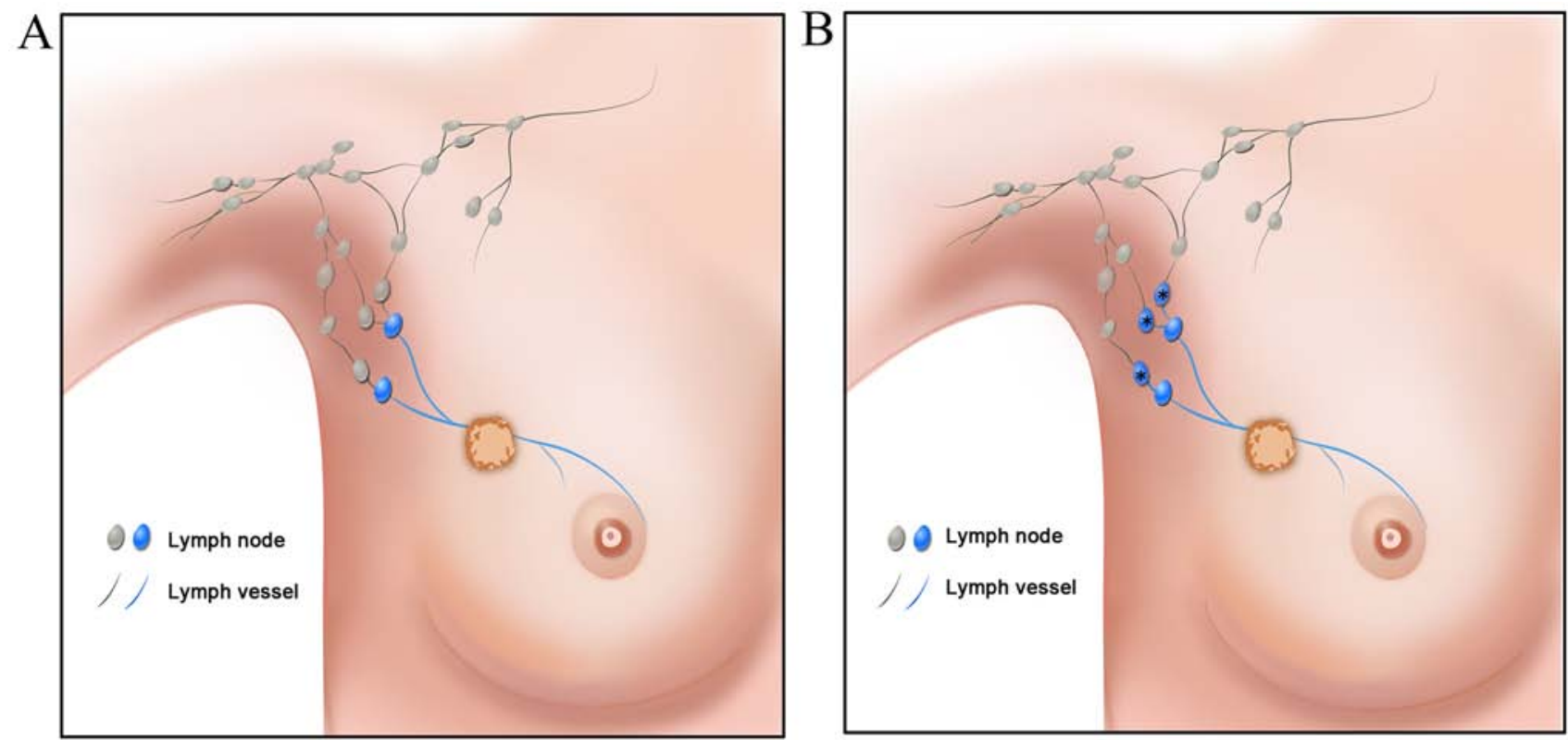

Figure 1. Pattern of SLNs and poSLNs in the breast. (A) Blue stained lymph nodes indicate SLNs. (B) Blue stained lymph nodes indicate SLNs and the asterisk-marked blue stained lymph nodes indicate poSLNs. SLNs, sentinel lymph nodes; poSLNs, post-sentinel lymph nodes.
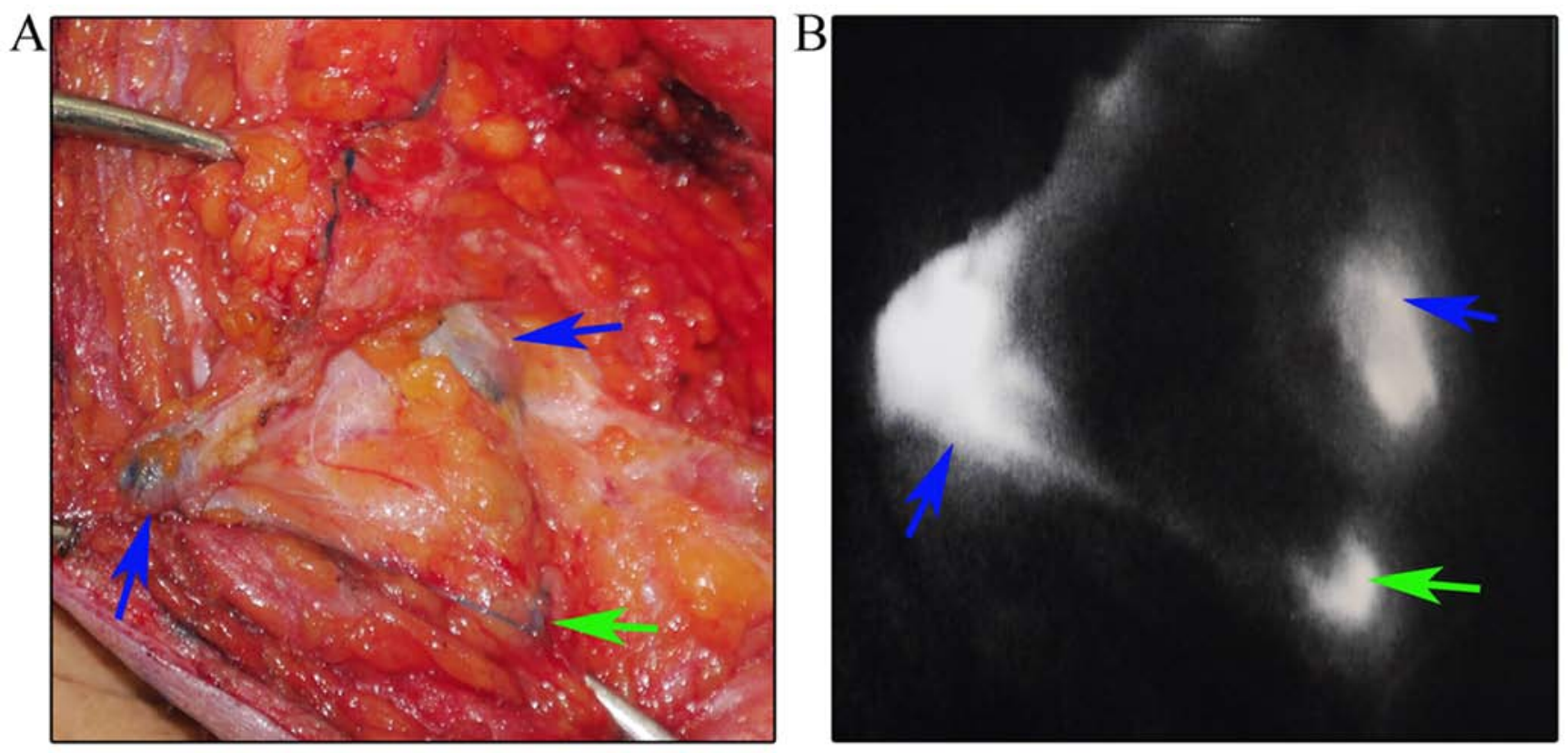

Figure 2. Representative images of SLNs with poSLNs in breast cancer during surgery. Blue arrows indicate SLNs and green arrows indicate poSLNs. (A) Dissection of the total lymphatic vessels with SLNs and poSLNs. (B) Fluorescent images of part (A) on the monitor screen of the fluorescence imaging system. Blue arrows are indicative of SLNs and green arrows of poSLNs. SLNs, sentinel lymph nodes; poSLNs, post-sentinel lymph nodes.

Initially, a total of 61 patients with breast cancer were enrolled. The characteristics of the patients are presented in Table I. Each patient received precise SLNB guided by the lymphatic drainage pathway. All lymph nodes that received the first lymphatic drainage were designated as SLNs. All SLNs could be stained. In addition, the lymphatic duct and poSLNs could be stained (Fig. 3). The results of the present study revealed that 30 patients exhibited 1 SLN, 17 patients 2 SLNs, 10 patients 3 SLNs, 1 patient 4 SLNs and 3 patients 5 SLNs. The median number of SLNs was 1.85 . A median of 2.28 poSLNs were resected. A total of $1,2,3,4,5$ and
6 poSLNs were identified in 23, 21, 6, 7, 3 and 1 patient(s), respectively (data not shown).

PoSLN dissection is not necessary in patients undergoing $S L N B$. The results of the present study revealed that 16 patients exhibited positive SLNs and 45 patients indicated negative SLNs. In the SLN-negative patients, all poSLNs were negative. Furthermore, no additional metastases were present in any of the axillary lymph nodes. In the 16 SLN-positive patients, 11 exhibited no additional lymph node metastasis, 2 presented with both positive SLNs 
A

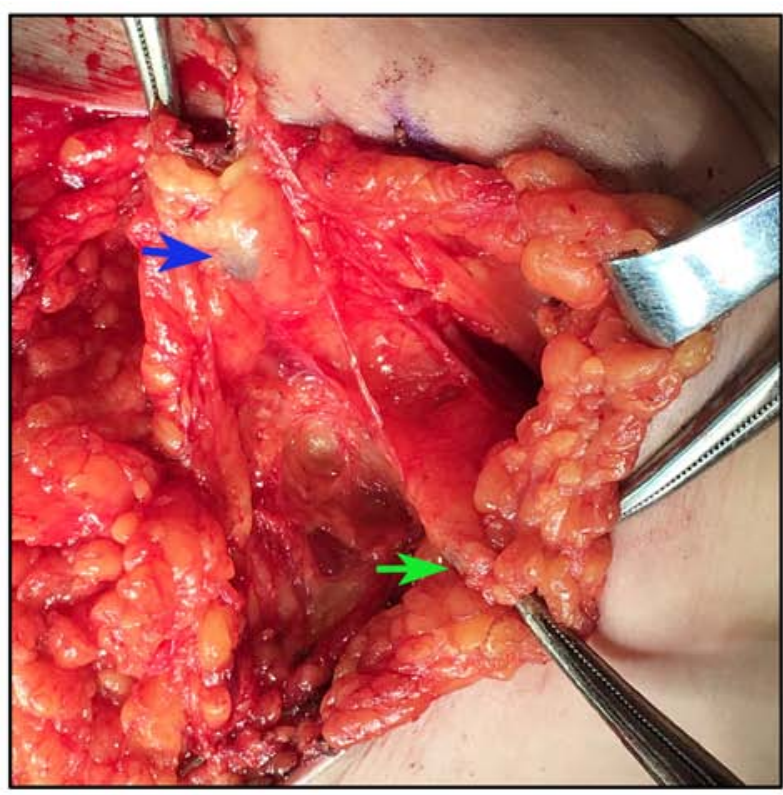

$\mathrm{C}$

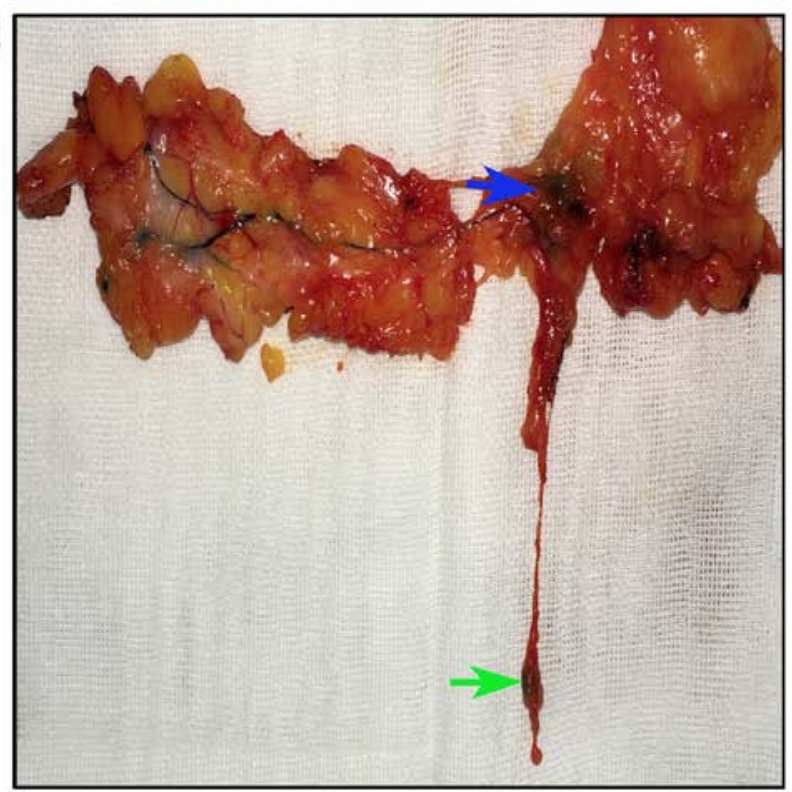

B

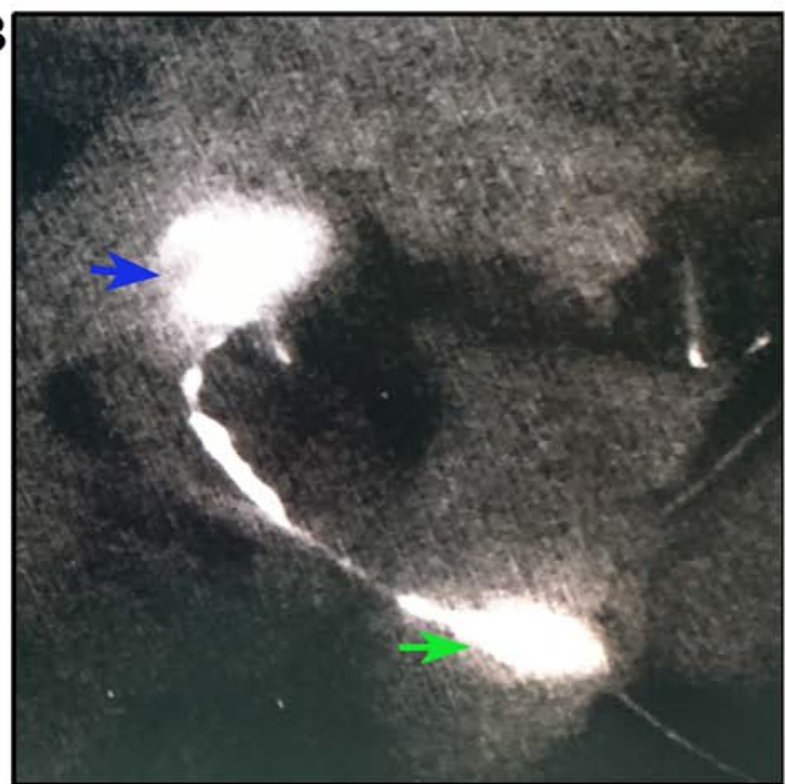

$\mathrm{D}$

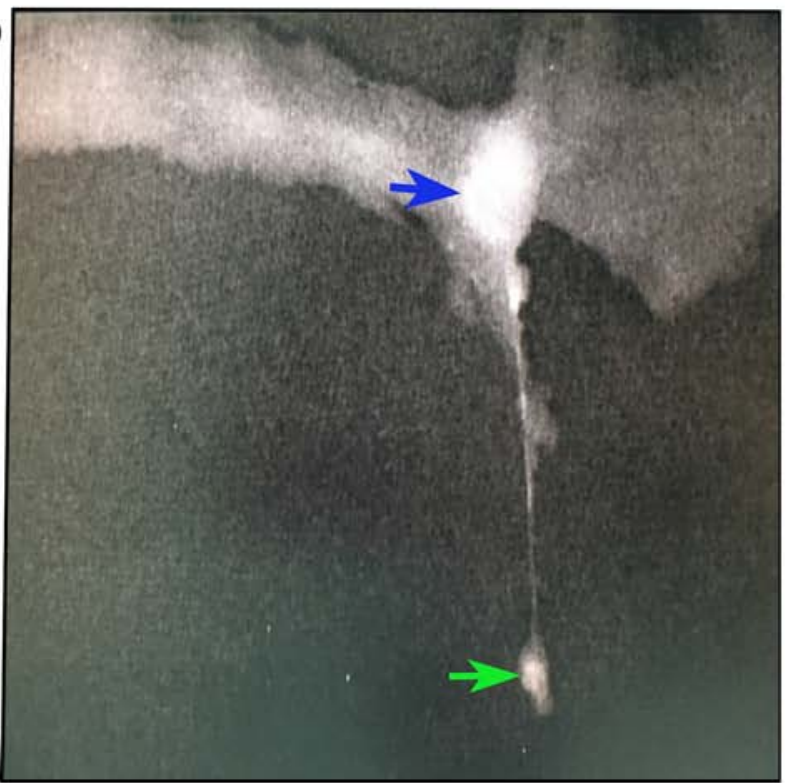

Figure 3. Identification of SLNs and poSLNs by precise SLNB. (A) Dissection of the total lymphatic vessels with SLNs and poSLNs. (B) Fuorescent images of part (A) on the monitor screen of the fluorescence imaging system. (C) Dissection of the total lymphatic vessels with SLNs and poSLNs. (D) Fluorescent images of part $(\mathrm{C})$ on the monitor screen of the fluorescence imaging system. Blue arrows are indicative of SLNs and green arrows of poSLNs. SLNs, sentinel lymph nodes; poSLNs, post-sentinel lymph nodes; SLNB, sentinel lymph node biopsy.

and poSLNs, and 3 had additional axillary lymph node metastasis (data not shown).

\section{Discussion}

Breast cancer is a frequently encountered cancer and is the second-leading cause of cancer-associated mortality worldwide in 2019 (28). James et al (29) reported 268,670 expected breast cancer cases and 41,400 expected deaths due to breast cancer in 2018 in the USA. Recurrence and metastasis are major causes of breast cancer-associated death (30). The assessment of the regional lymph node status in patients with breast cancer is important for the treatment and prediction of prognosis (31). ALND is used to assess regional lymph node status in patients with breast cancer. However, it appears to be associated with increased morbidity of complications including arm lymphedema and pain, seroma formation and vascular and brachial plexus injuries $(32,33)$. SLNB has rapidly been replacing standard axillary lymph dissection as the choice of several surgeons, and it is considered the standard procedure for axillary evaluation in early-stage breast cancer $(2,34)$.

With regard to the development of SLNB, less aggressive axillary surgery is applied in breast cancer; however, surgeon bias may exist during the procedure (35). Therefore, it is vital to conduct SLNB precisely. In the present study, a novel method was developed for intraoperative, precise SLNB in patients with breast cancer. The data indicated that the current method exhibited high sensitivity. To the best of our knowledge, the present study is the first to introduce the concept of poSLNs. This novel method can precisely identify true SLNs and 
stained non-SLNs. The number of SLNs was time-dependent, and the results were consistent with those reported in previous studies $(12,16,18-22)$. Therefore, it is not appropriate to regard all blue stained or fluorescence-positive lymph nodes as SLNs. In addition, as the number of SLNs for patients is uncertain, it is not appropriate to remove at least 2 or $4-5$ lymph nodes during SLNB. Therefore, the identification and preservation of stained non-SLNs in breast cancer is important.

The clinical studies that have been performed on SLNB have mostly focused on accuracy, detection rates and false negative rates $(8,11,12)$. Therefore, surgeons may remove a higher number of non-SLNs and designate them as SLNs. This approach may reduce the false negative rate, while increasing potential complications. Previous studies have reported that the number of SLNs varies between 1 and $12(21,36,37)$. In addition, the number of SLNs identified in a combined group (ICG and $\mathrm{MB}$ ) has been shown to be higher than that found in the blue dye or RI alone groups $(38,39)$. This finding demonstrated a risk in detecting specific non-SLNs. In the present study, all patients were negative for poSLNs in the SLN-negative group. No additional metastases were noted in the axillary lymph nodes. The data confirmed that the duration of time following ICG and MB injection was highly significant for SLNB. The present study strongly suggested that the resection of poSLNs was not necessary when SLNB was conducted. In addition, two patients exhibited no additional lymph node metastases, with the exception of poSLNs and SLNs. The Z0011 trial suggested that avoidance of an ALND may be permitted, even for SLN-positive patients (37). This finding suggests that a proportion of patients with positive SLNs may avoid ALND if poSLNs are removed in breast cancer patients undergoing SLNB. The status of both SLNs and poSLNs may be superior to that of SLNs alone during the assessment of the axillary lymph node status. The combination of SLNB and poSLNB may provide a substitute for ALND in certain SLN-positive patients, which should be tested in future studies.

The present study contains certain limitations. Firstly, the patient number was relatively small. Secondly, the medical information lacked data on disease prognosis and associated complications of these patients. Therefore, a large randomized, controlled, multicenter trial should be conducted in the future in order to address these limitations.

In conclusion, the present study demonstrated that intraoperative dissection of the lymphatic network can distinguish true SLNs and stained non-SLNs, such as poSLNs. The data confirmed that not all stained lymph nodes were true SLNs. Furthermore, it was shown that identification and preservation of stained non-SLNs is possible during SLNB in breast cancer. The current study provides important clinical applications for precision medicine.

\section{Acknowledgements}

Not applicable.

\section{Funding}

This work was supported by the National Natural Science Foundation of China (grant nos. 81672613 and 81874119), the Special Foundation for Taishan Scholars (grant no. ts20190971), the Special Support Plan for National High Level Talents (Ten Thousand Talents Program W01020103), the National Key Research and Development Program (grant no. 2018YFC0114705) and the Qilu Hospital Clinical New Technology Developing Foundation (grant nos. 2018-7 and 2019-3).

\section{Availability of data and materials}

The datasets used and/or analyzed during the current study are available from the corresponding author on reasonable request.

\section{Authors' contributions}

QY and XL designed the study and drafted the manuscript. $\mathrm{XL}, \mathrm{SC}$ and YD were involved in acquisition of data, drafting and revising the manuscript. HG, LJ, XK and TM edited the manuscript and were involved in the conception and design of the study. All authors read and approved the final manuscript.

\section{Ethics approval and consent to participate}

All procedures performed in the present study were in accordance with the ethical standards of the institutional and/or national research committee and with the 1964 Helsinki Declaration and its later amendments or comparable ethical standards. The study was approved by the Ethics Committee on Scientific Research of Shandong University Qilu Hospital (Jinan, China; approval no. KYLL-2016-231). Written informed consent was obtained from all patients.

\section{Patient consent for publication}

Not applicable.

\section{Competing interests}

The authors declare that they have no competing interests.

\section{References}

1. Manca G, Tardelli E, Rubello D, Gennaro M, Marzola MC, Cook GJ and Volterrani D: Sentinel lymph node biopsy in breast cancer: A technical and clinical appraisal. Nucl Med Commun 37: 570-576, 2016.

2. Lyman GH, Temin S, Edge SB, Newman LA, Turner RR, WeaverDL, Benson AB III, Bosserman LD, Burstein HJ, Cody H III, et al: Sentinel lymph node biopsy for patients with early-stage breast cancer: American society of clinical oncology clinical practice guideline update. J Clin Oncol 32: 1365-1383, 2014.

3. Vuthaluru S and Srivastava A: Axillary vs. sentinel lymph node dissection for invasive breast cancer. JAMA 305: 2290, 2011.

4. Lyman GH, Somerfield MR, Bosserman LD, Perkins CL, Weaver DL and Giuliano AE: Sentinel lymph node biopsy for patients with early-stage breast cancer: American society of clinical oncology clinical practice guideline update. J Clin Oncol 35: 561-564, 2017.

5. Yi M, Meric-Bernstam F, Ross MI, Akins JS, Hwang RF, Lucci A, Kuerer HM, Babiera GV, Gilcrease MZ and Hunt KK: How many sentinel lymph nodes are enough during sentinel lymph node dissection for breast cancer? Cancer 113: 30-37, 2008.

6. Kim MK, Park HS, Kim JY, Kim S, Nam S, Park S and Kim SI: The clinical implication of the number of lymph nodes harvested during sentinel lymph node biopsy and its effects on survival outcome in patients with node-negative breast cancer. Am J Surg 214: 726-732, 2017. 
7. Boughey JC, Ballman KV, Hunt KK, McCall LM, Mittendorf EA, Ahrendt GM, Wilke LG and Le-Petross HT: Axillary ultrasound after neoadjuvant chemotherapy and its impact on sentinel lymph node surgery: Results from the American college of surgeons oncology group Z1071 trial (alliance). J Clin Oncol 33: 3386-3393, 2015.

8. Boileau JF, Poirier B, Basik M, Holloway CMB, Gaboury L, Sideris L, Meterissian S, Arnaout A, Brackstone M, McCready DR, et al: Sentinel node biopsy after neoadjuvant chemotherapy in biopsy-proven node-positive breast cancer: The SN FNAC study. J Clin Oncol 33: 258-264, 2015.

9. Zakaria S, Degnim AC, Kleer CG, Diehl KA, Cimmino VM, Chang AE, Newman LA and Sabel MS: Sentinel lymph node biopsy for breast cancer: How many nodes are enough? J Surg Oncol 96: 554-559, 2007.

10. Liedtke C, Jackisch C, Thill M, Thomssen C, Müller V, Janni W and AGO Breast Committee: AGO recommendations for the diagnosis and treatment of patients with early breast cancer: Update 2018. Breast Care (Basel) 13: 196-208, 2018.

11. Sugie T, Kinoshita T, Masuda N, Sawada T, Yamauchi A, Kuroi K Taguchi T, Bando H, Yamashiro H, Lee T, et al: Evaluation of the clinical utility of the ICG fluorescence method compared with the radioisotope method for sentinel lymph node biopsy in breast cancer. Ann Surg Oncol 23: 44-50, 2016.

12. Inoue T, Nishi T, Nakano Y, Nishimae A, Sawai Y, Yamasaki M and Inaji $\mathrm{H}$ : Axillary lymph node recurrence after sentinel lymph node biopsy performed using a combination of indocyanine green fluorescence and the blue dye method in early breast cancer. Breast Cancer 23: 295-300, 2016.

13. Lorek A, Boratyn-Nowicka A and Szlachta-Światkowska E: Sentinel lymph node (sln) in breast cancer. Review of current identification methods. Wiad Lek 70: 85-91, 2017.

14. Percy DB, Pao JS, McKevitt E, Dingee C, Kuusk U and Warburton R: Number of nodes in sentinel lymph node biopsy for breast cancer: Are surgeons still biased? J Surg Oncol 117: 1487-1492, 2018.

15. Shen S, Xu Q, Zhou Y, Mao F, Guan J and Sun Q: Comparison of sentinel lymph node biopsy guided by blue dye with or without indocyanine green in early breast cancer. J Surg Oncol 117: $1841-1847,2018$

16. Tong $\mathrm{M}$, Guo $\mathrm{W}$ and Gao $\mathrm{W}$ : Use of fluorescence imaging in combination with patent blue dye versus patent blue dye alone in sentinel lymph node biopsy in breast cancer. J Breast Cancer 17: 250-255, 2014

17. Hirano A, Kamimura M, Ogura K, Kim N, Hattori A, Setoguchi Y, Okubo F, Inoue H, Miyamoto R, Kinoshita J, et al: A comparison of indocyanine green fluorescence imaging plus blue dye and blue dye alone for sentinel node navigation surgery in breast cancer patients. Ann Surg Oncol 19 4112-4116, 2012.

18. Papadia A, Gasparri ML, Buda A and Mueller MD: Sentinel lymph node mapping in endometrial cancer: Comparison of fluorescence dye with traditional radiocolloid and blue. J Cancer Res Clin Oncol 143: 2039-2048, 2017.

19. Pitsinis V, Provenzano E, Kaklamanis L, Wishart GC and Benson JR: Indocyanine green fluorescence mapping for sentinel lymph node biopsy in early breast cancer. Surg Oncol 24 375-379, 2015

20. van der Vorst JR, Schaafsma BE, Verbeek FP, Hutteman M, Mieog JSD, Lowik CWG, Liefers GJ, Frangioni JV, van de Velde CJH and Vahrmeijer AL: Randomized comparison of near-infrared fluorescence imaging using indocyanine green and $99(\mathrm{~m})$ technetium with or without patent blue for the sentinel lymph node procedure in breast cancer patients. Ann Surg Oncol 19: 4104-4111, 2012.

21. Hirche C, Murawa D, Mohr Z, Kneif S and Hunerbein M: ICG fluorescence-guided sentinel node biopsy for axillary nodal staging in breast cancer. Breast Cancer Res Treat 121: 373-378, 2010.

22. Toh U, Iwakuma N, Mishima M, Okabe M, Nakagawa S and Akagi Y: Navigation surgery for intraoperative sentinel lymph node detection using Indocyanine green (ICG) fluorescence real-time imaging in breast cancer. Breast Cancer Res Treat 153 $337-344,2015$
23. Cserni G, Chmielik E, Cserni B and Tot T: The new TNM-based staging of breast cancer. Virchows Arch 472: 697-703, 2018.

24. Li X, Chen S, Jiang L, Kong X, Ma T, Xu H and Yang Q: Precise intraoperative sentinel lymph node biopsies guided by lymphatic drainage in breast cancer. Oncotarget 8: 63064-63072, 2017.

25. Su P, Zhang Q and Yang Q: Immunohistochemical analysis of Metadherin in proliferative and cancerous breast tissue. Diagn Pathol 5: 38, 2010.

26. Zhang N, Li X, Tao K, Jiang L, Ma T, Yan S, Yuan C, Moran MS, Liang F, Haffty BG and Yang Q: BCL-2 (-938C > A) polymorphism is associated with breast cancer susceptibility. BMC Med Genet 12: 48, 2011.

27. Zhang X, Li Y, Zhou Y, Mao F, Lin Y, Guan J and Sun Q: Diagnostic performance of indocyanine green-guided sentinel lymph node biopsy in breast cancer: A meta-analysis. PLoS One 11: e0155597, 2016.

28. DeSantis CE, Ma J, Gaudet MM, Newman LA, Miller KD, Sauer AG, Jemal A and Siegel RL: Breast cancer statistics, 2019. CA Cancer J Clin 69: 438-451, 2019.

29. James TA, Coffman AR, Chagpar AB, Boughey JC, Klimberg VS, Morrow M, Giuliano AE and Harlow SP: Troubleshooting sentinel lymph node biopsy in breast cancer surgery. Ann Surg Oncol 23: 3459-3466, 2016.

30. Valastyan S and Weinberg RA: Tumor metastasis: Molecular insights and evolving paradigms. Cell 147: 275-292, 2011.

31. Gerber B, Heintze K, Stubert J, Dieterich M, Hartmann S, Stachs A and Reimer T: Axillary lymph node dissection in early-stage invasive breast cancer: Is it still standard today? Breast Cancer Res Treat 128: 613-624, 2011.

32. Mansel RE, Fallowfield L, Kissin M, Goyal A, Newcombe RG, Dixon JM, Yiangou C, Horgan K, Bundred N, Monypenny I, et al: Randomized multicenter trial of sentinel node biopsy versus standard axillary treatment in operable breast cancer: The ALMANAC trial. J Natl Cancer Inst 98: 599-609, 2006.

33. Kim T, Giuliano AE and Lyman GH: Lymphatic mapping and sentinel lymph node biopsy in early-stage breast carcinoma: A metaanalysis. Cancer 106: 4-16, 2006.

34. Giuliano AE, Ballman KV, McCall L, Beitsch PD, Brennan MB, Kelemen PR, Ollila DW, Hansen NM, Whitworth PW, Blumencranz PW, et al: Effect of axillary dissection vs. no axillary dissection on 10-year overall survival among women with invasive breast cancer and sentinel node metastasis: The ACOSOG Z0011 (alliance) randomized clinical trial. JAMA 318: 918-926, 2017.

35. Dixon JM, Grewar J, Twelves D, Graham A, Martinez-Perez C and Turnbull A: Factors affecting the number of sentinel lymph nodes removed in patients having surgery for breast cancer. Breast Cancer Res Treat, Aug 18, 2020 (Online ahead of print).

36. Aoyama K, Kamio T, Ohchi T, Nishizawa M and Kameoka S: Sentinel lymph node biopsy for breast cancer patients using fluorescence navigation with indocyanine green. World J Surg Oncol 9: 157, 2011.

37. Giuliano AE, Hunt KK, Ballman KV, Beitsch PD, Whitworth PW, Blumencranz PW, Leitch AM, Saha S, McCall LM and Morrow M: Axillary dissection vs. no axillary dissection in women with invasive breast cancer and sentinel node metastasis: A randomized clinical trial. JAMA 305: 569-575, 2011.

38. Ji Y, Luo N, Jiang Y, Li Q, Wei W, Yang H and Liu J: Clinical utility of the additional use of blue dye for indocyanine green for sentinel node biopsy in breast cancer. J Surg Res 215: 88-92, 2017.

39. Sugie T, Sawada T, Tagaya N, Kinoshita T, Yamagami K, Suwa H, Ikeda T, Yoshimura K, Niimi M, Shimizu A and Toi M: Comparison of the indocyanine green fluorescence and blue dye methods in detection of sentinel lymph nodes in early-stage breast cancer. Ann Surg Oncol 20: 2213-2218, 2013.

This work is licensed under a Creative Commons Attribution-NonCommercial-NoDerivatives 4.0 International (CC BY-NC-ND 4.0) License. 